\title{
A MICROMACHINED VARIABLE CAPACITOR FOR MONOLITHIC LOW-NOISE VCOS
}

\author{
Darrin J. Young, Bernhard E. Boser \\ Department of EECS, University of California \\ Berkeley, California 94720
}

\begin{abstract}
An aluminum micromachined variable capacitor is proposed as the tuning element in voltage-controlled oscillators (VCOs) in applications such as cellular phones. Compared to the traditional solutions relying on varactor diodes, this approach is amenable to monolithic integration in a standard electronic circuit process without sacrificing performance. An experimental device consisting of four parallel microstructures is tunable between $2.11 \mathrm{pF}$ and $2.46 \mathrm{pF}$ with a $5.5 \mathrm{~V}$ DC tuning voltage. At $1 \mathrm{GHz}$, the quality factor $(\mathrm{Q})$ is 62 , limited by the resistive loss in the interconnect traces.
\end{abstract}

\section{INTRODUCTION}

Increased demand for wireless communication motivates a growing interest in monolithic all-CMOS personal communication transceivers [1]. Sub-micron technologies meet RF circuit speed requirements, but still depend on off-chip components to implement some key building blocks. Off-chip devices result in increased fabrication cost and power dissipation, and reduced reliability.

The various cellular phone standards, for example, require VCOs with frequencies in the low Gigahertz range and a tuning range of 50 to $100 \mathrm{MHz}$. Channel spacing of only $30 \mathrm{KHz}$ calls for extremely low phase-noise of the oscillator. Monolithic solutions based on ring oscillators [2] cannot meet this requirement. Since the phase-noise exhibited by this type of oscillator is a function of power dissipation rather than electronic circuit speed, little improvement can be expected from future technology scaling.

Current VCO designs in personal communication devices rely on an off-chip LC tank circuit with a $Q$ of at least 10 to meet the low phase-noise requirements. Typical element values are on the order of $2 \mathrm{pF}$ for the capacitor, and $6 \mathrm{nH}$ for the inductor. Frequency tuning is achieved by modulating the depletion width of a varactor diode. Unfortunately, this solution has been proven difficult to realize in monolithic form. While in recent years substantial progress has been made to improve the quality of on-chip spiral inductors, high-quality varactor diodes require special materials that are not available in standard IC processes and hence are not suited for monolithic integration. Silicon diodes suffer both from an excessive series loss and hence low quality factor, and from a limited tuning range [3].

In this paper, a new solution based on a micromachined parallel-plate variable capacitor is proposed. The device consists of a thin sheet of aluminum suspended in air approximately $1.5 \mu \mathrm{m}$ above the substrate. A DC voltage results in an electrostatic force pulling the movable plate closer to the substrate, hence increasing the capacitance. Theoretically, a tuning range of up to $50 \%$ can be achieved. The required tuning voltage can be chosen by appropriately dimensioning the suspension. This represents an important advantage over varactor diodes which have a tuning range that is a strong function of the supply voltage. For a typical $3 \mathrm{~V}$ maximum tuning voltage, the adjustment range of varactor diodes usually can- not cover both the required oscillator tuning range plus typical manufacturing tolerances; hence, it requires (manual) tuning. Compared to varactor-based oscillators, a larger oscillation amplitude is possible with micromachined capacitors for two reasons. First, unlike the varactor, the proposed capacitor does not have the potential problem of becoming forward-biased. Second, it does not suffer from high-frequency distortion because of its mechanical damping.

Despite the better mechanical properties and the vast experience with polysilicon microstructure fabrication [4], aluminum [5, 6] is used as the structural material in this work for several reasons. First, its lower sheet resistance is critical to minimize ohmic losses and guarantee an adequate quality factor. The second key advantage of aluminum is the low processing temperature of only $150^{\circ} \mathrm{C}$ for the proposed fabrication procedure. Because of this, the variable capacitors can be fabricated on top of wafers with completed electronic circuits without affecting device characteristics. This is particularly crucial in applications such as RF where the availability of the most recent IC technology is a critically competitive advantage. The inferior mechanical properties of aluminum compared to polysilicon are not critical for this application.

\section{MICROMACHINED VARIABLE CAPACITOR}

Figure 1 shows top and cross-sectional views of the variable capacitor. It consists of a thin aluminum plate suspended in air nominally $1.5 \mu \mathrm{m}$ above a bottom aluminum layer and anchored with four mechanical folded-beam suspensions acting as springs. The folding relaxes the stress in the film and thus reduces the warpage [7]. The symmetry also avoids systematic tilt of the structure. The warping is mainly caused by the thermal expansion mismatch between the sacrificial material (photoresist) and the top aluminum layer. The elevated temperature during the deposition and subsequent cooling to the room temperature further contributes to a built-in strain gradient in the aluminum film and causes the structure to warp after removal of the sacrificial layer.

The chosen plate size of 200 by $200 \mu \mathrm{m}^{2}$ and $1.5 \mu \mathrm{m}$ nominal air-gap result in a nominal capacitance value of approximately 200fF. These dimensions represent a compromise between the achievable capacitance value and the maximum structure size that can be fabricated reliably without excessive warping. Difficulties in removing the sacrificial material preclude a smaller air-gap despite the potential for higher capacitance density and hence smaller overall size. The achieved dimensions present a substantial advance for micromachined aluminum structures. Previously reported results describe much smaller structures with larger gap size $[5,6]$.

A DC bias voltage applied across the capacitor results in an electrostatic pull-down force and consequent reduction of the airgap. The suspended plate can be pulled down at most by $1 / 3$ of the original gap size before the pull-down force exceeds the mechanical restoring force causing the plate to be pulled all the way to the sub- 
strate. This corresponds to an up to $50 \%$ increase in capacitance value.

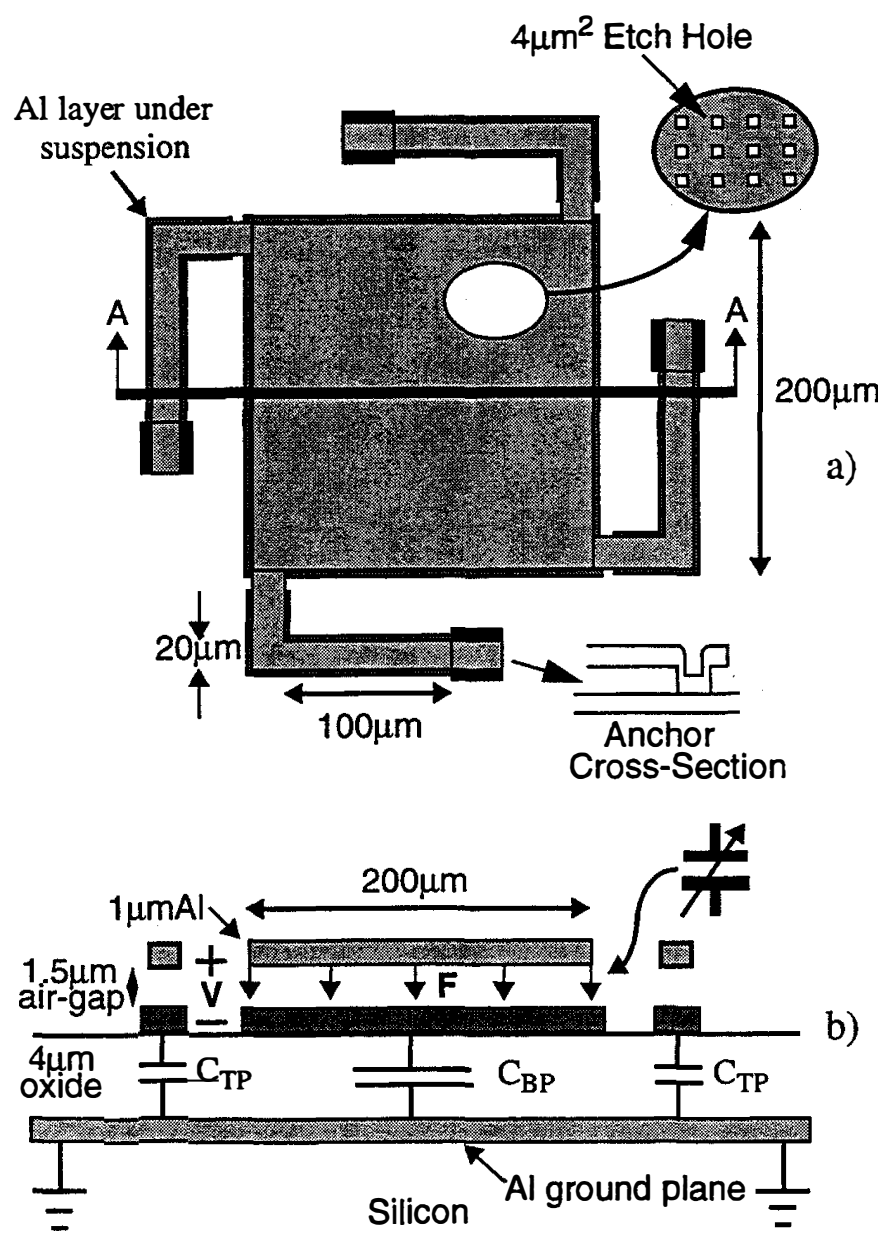

Figure 1. a) Top view, b) cross-section of a micromachined variable capacitor

In RF transceivers, the DC tuning voltage is typically limited to $3 \mathrm{~V}$ or less by the supply voltage. Assuming a $1.5 \mu \mathrm{m}$ nominal gap size and $80 \mathrm{ng}$ mass of the 200 by $200 \mu \mathrm{m}^{2}$ plate, this calls for a suspension spring constant of $3.8 \mathrm{~N} / \mathrm{m}$ and corresponds to a resonant frequency of $35 \mathrm{KHz}$. The suspension consists of four beams of $100 \mu \mathrm{m}$ length and $20 \mu \mathrm{m}$ width.

To demonstrate the concept, four variable capacitors are wired in parallel to obtain a $400 \mathrm{fF}$ tuning range and a $800 \mathrm{fF}$ nominal capacitance (Figure 5). The actual overall capacitance is larger because of fixed parasitics. A Q of at least 40 at $1 \mathrm{GHz}$ is needed to achieve a similar performance as a discrete varactor diode. This corresponds to a total series resistive loss of less than $2 \Omega$.

Figure $1 \mathrm{~b}$ shows the parasitics capacitances, $\mathrm{C}_{\mathrm{TP}}$ and $\mathrm{C}_{\mathrm{BP}}$ between the top and bottom plates of the variable capacitor and the substrate. In a VCO application, the bottom plate is grounded and $\mathrm{C}_{\mathrm{BP}}$ is therefore shorted. However, the top-plate parasitics, $\mathrm{C}_{\mathrm{TP}}$, appear in parallel with the variable capacitor. This not only reduces the tuning range, but can also lower the quality factor of the overall capacitor. These problems are alleviated with a separated aluminum layer directly on the silicon substrate isolated from the capacitor bottom-plate with a $4 \mu \mathrm{m}$ thick oxide. The thick oxide minimizes the value of the parasitic capacitance. The aluminum layer shields the parasitics from the lossy substrate, thus ensuring a high Q. Possibly the aluminum ground plane could be replaced with an interconnect aluminum layer to eliminate some fabrication steps.

In this design the bottom-plate aluminum extends from the anchor under the suspension up to the edge of the movable plate. This conservative design has been chosen to prevent the capacitors from shorting out when the suspension touches the bottom plate. However, it results in a relatively large parasitic capacitance of $220 \mathrm{fF}$ for all four suspensions and substantially reduces the tuning range. The capacitor shorting has been found not to be a problem in experimental devices. In the next version, the layout shown in Figure 2 will be used, resulting in an increase of the variable capacitance by about $100 \mathrm{fF}$ and a decrease of $\mathrm{C}_{\mathrm{TP}}$ to $70 \mathrm{fF}$. The interconnect traces of the four individual capacitors shown in Figure 5 add approximately $280 \mathrm{fF}$ parasitic capacitance and $0.7 \Omega$ and $0.3 \Omega$ series resistance to the wiring of the top and bottom plates respectively.

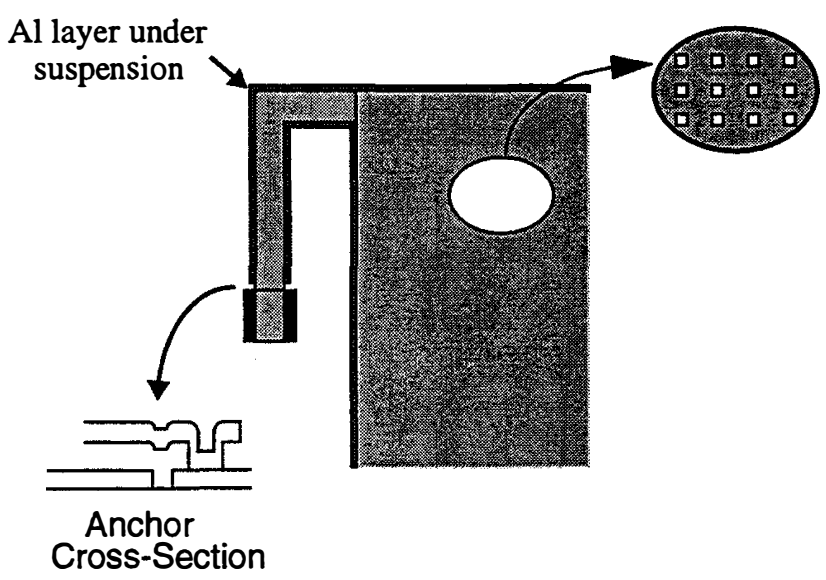

Figure 2. Suggested new layout

\section{FABRICATION PROCESS}

To experiment with the processing, the variable capacitor has first been fabricated on a bare silicon wafer. Fabrication on top of completed electronic circuits is planned as a next step.

Figure 3 illustrates the process flow. First, a $1 \mu \mathrm{m}$ thick aluminum film is sputtered onto the bare silicon wafer to form a lowresistance ground plane. Next, a $4 \mu \mathrm{m}$ low-temperature oxide is deposited (Fig. 3a). The bottom plate of the capacitor and interconnect trace consist of another $1 \mu \mathrm{m}$ thick sputtered aluminum film (Fig. 3b). Wet etching is chosen to pattem this layer since the lateral dimensions of the structures are not critical. A $1.5 \mu \mathrm{m}$ thick photoresist serves as a sacrificial layer. This material offers excellent control of thickness and uniformity, is photo-definable and easy to process. Contact windows to the bottom aluminum are opened in the resist (Fig. 3c). Then it is baked at $120^{\circ} \mathrm{C}$ for 45 minutes to prevent out-gassing during the subsequent deposition of the top aluminum layer. Longer baking time would result in hardening of the resist and consequent difficulties in its removal. The final $1 \mu \mathrm{m}$ aluminum layer is sputtered on at a reduced power and also wet etched since a plasma dry etch would cause the sacrificial layer to outgas. This layer forms anchors to the bottom aluminum inside the contact windows through the resist (Fig. 3d). In addition, it is deposited on all interconnects to lower the resistance (not shown in the figure). Etch holes of 2 by $2 \mu \mathrm{m}^{2}$ size spaced $10 \mu \mathrm{m}$ apart ensure a complete removal of the sacrificial photoresist. 
At this point, the wafers are coated with photoresist to protect against particulates during dicing. After dicing and removal of particulates, the protective resist and sacrificial layer are removed with an oxygen-based dry etch, thus releasing the capacitor top-plate (Fig. 3e).

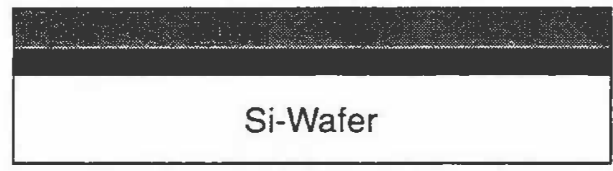

LTO

AL1

a)

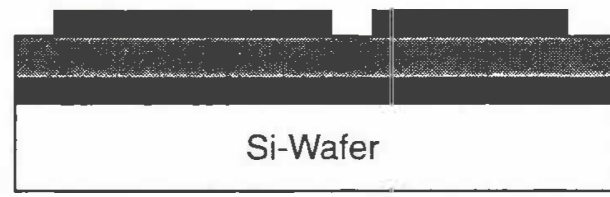

AL2

LTO

AL1

b)

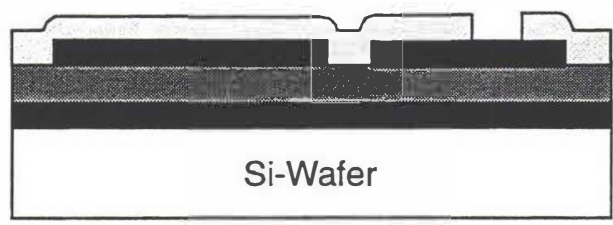

Photoresist

AL2

LTO

AL1

c)

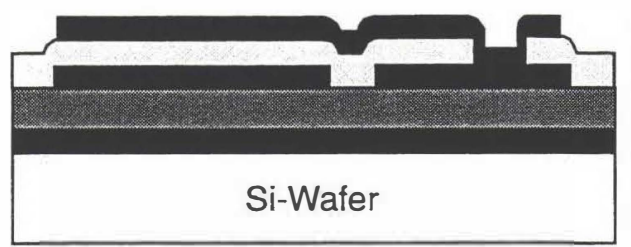

AL3

Photoresist

AL2

LTO

AL1

d)

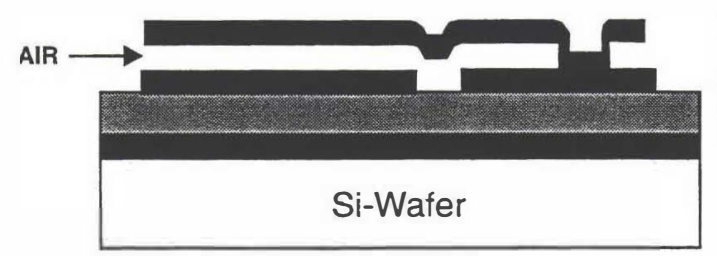

AL3

Photoresist

AL2

LTO

AL1

e)

Figure 3. Process flow of the post-CMOS

$\mathrm{Al}$ micromachining technology:

a) ground plane ( $1 \mu \mathrm{m}$ aluminum) and LTO deposition,

b) first aluminum layer deposition $(1 \mu \mathrm{m})$ and patterning,

c) photoresist sacrificial layer $(1.5 \mu \mathrm{m})$,

d) second aluminum layer $(1 \mu \mathrm{m})$ with anchor,

e) released structure after etching photoresist.

The oxygen gas pressure and plasma power must be set carefully to ensure a complete removal of the resist and prevent the aluminum film from warping significantly. The structure heats up to $150^{\circ} \mathrm{C}$ during the 180 -minute long etch. An optimal pressure of $500 \mathrm{mT}$ and $150 \mathrm{~W}$ power have been determined experimentally. The dry etching process virtually eliminates the problem of the structure sticking to the substrate. This is an important advantage over the wet release procedure typically used with polysilicon microstructures.

\section{EXPERIMENT RESULTS}

Figure 4 shows SEM micrographs of a single tunable capacitor. Optical inspection reveals doming of the capacitor top-plate. At the center of the structure, the gap size is $0.5 \mu \mathrm{m}$ larger than the nominal value of $1.5 \mu \mathrm{m}$, and the comers are $0.3 \mu \mathrm{m}$ below the nominal height.

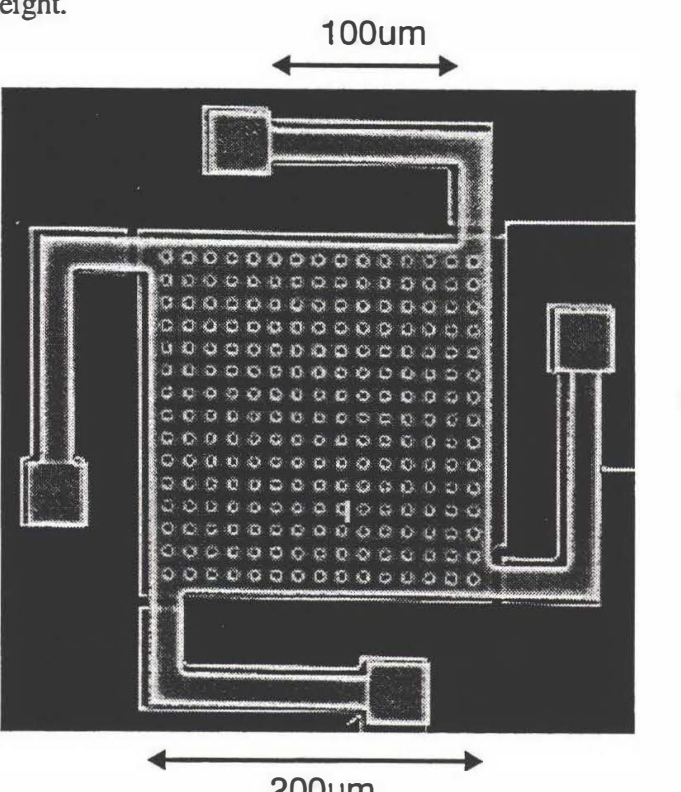

a)

b)

Figure 4. SEM of $200 \times 200 \mu \mathrm{m}^{2}$ micromachined aluminum tunable capacitor

a) Top view, b) Suspension detail

Figure 5 presents the parallel connection of four variable capacitor with a nominal capacitance value of $2 \mathrm{pF}$ designed for a

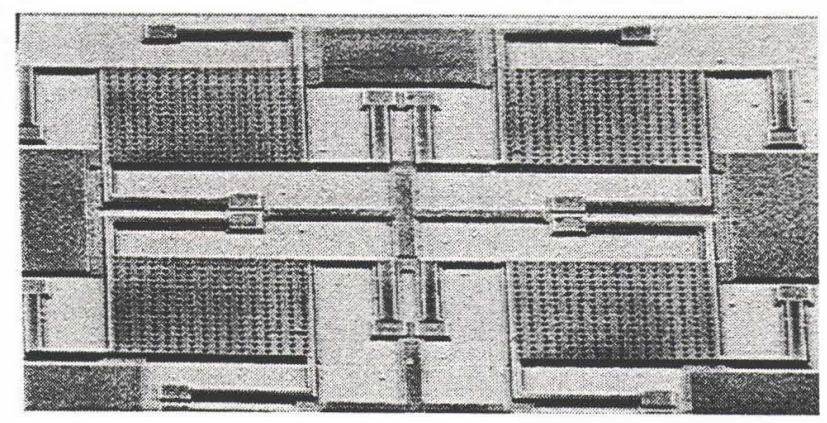

Figure 5. Four parallel microstructures 
experimental VCO. With a $5.5 \mathrm{~V}$ tuning voltage, the capacitance value can be varied between $2.11 \mathrm{pF}$ and $2.46 \mathrm{pF}$. This corresponds to a tuning range of $16 \%$ shown in Figure 6 . The tuning voltage is larger than the designed $3 \mathrm{~V}$ but can be lowered by decreasing the width of the suspension. The tuning range can be increased by appropriate layout changes as illustrated in Figure 2 and described above.

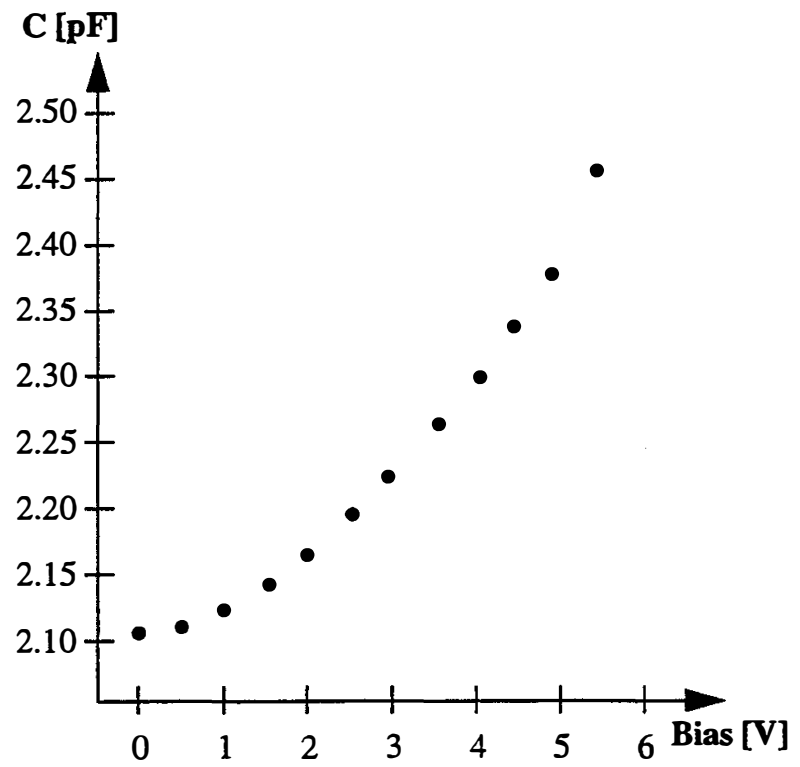

Figure 6. Measured capacitance as a function of the applied DC Bias

Aside from the capacitance, the quality factor is the most important electrical parameter of the structure. It is most accurately determined with an S-parameter measurement. Figure 7 shows the Smith Chart for the four parallel structures measured with an HP8719 network analyzer for frequency up to $1.2 \mathrm{GHz}$. The S11

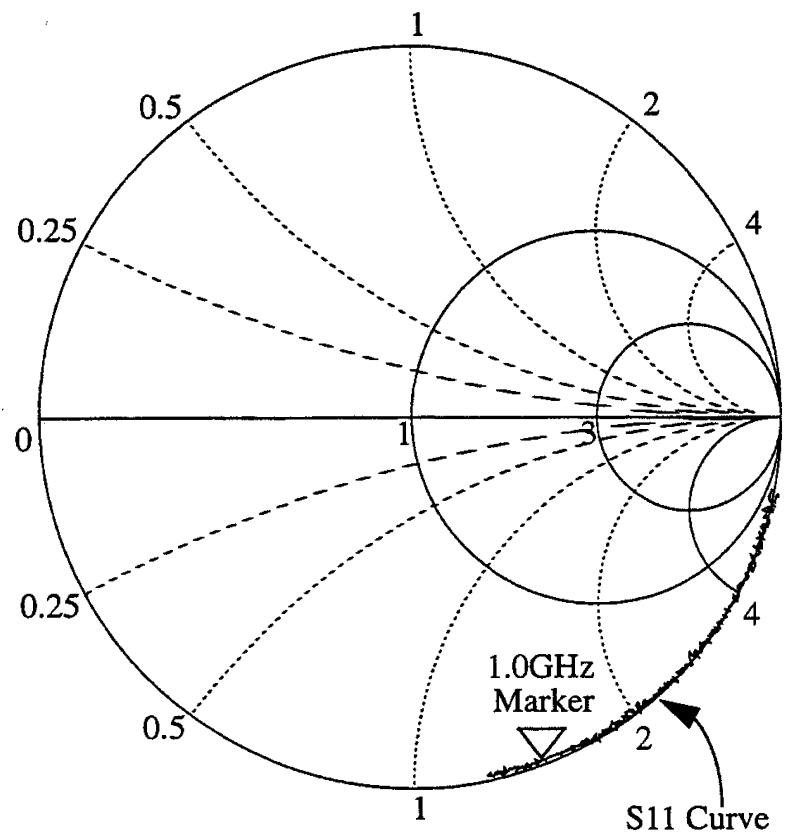

START: $0.1600 \mathrm{GHz}$, STOP: $1.200 \mathrm{GHz}$

Figure 7. Measured S11 in a Smith Chart curve follows closely the lower-half of the unit circle, as is expected for a capacitor. At $1 \mathrm{GHz}$, the series resistance is $1.2 \Omega$,. This value only slightly exceeds the projected wiring resistance and corresponding to a $Q$ of 62 . It matches or exceeds the quality factor of varactor diodes and is at least an order of magnitude larger than that of a typical junction capacitor implemented in a standard IC process.

\section{CONCLUSION}

Aluminum micromachined variable capacitors constitute an attractive alternative to conventional varactor diodes as the tuning element in voltage-controlled oscillators for personal communication devices. The proposed device meets or exceeds the performance of typical varactors and can be added to standard IC processes with a few simple back-end processing steps. Further advantages over a varactor-based solution include the possibility to operate at lower supply voltages and a larger oscillation amplitude.

Unlike polysilicon micromachining processes, this technology requires no changes of the underlying IC process, a feature that may be attractive also for other applications, for example inertial sensors. The performance of such sensors has not yet been determined.

\section{ACKNOWLEDGMENTS}

The authors thank James Bustillo from the Berkeley Sensor and Actuator Center for his help with device fabrication. All experiments have been carried out in the Berkeley Microfabrication Laboratory. Financial support from NSF is gratefully acknowledged.

\section{REFERENCES}

[1] P. R. Gray, R. G. Meyer, "Future Direction in Silicon ICs for RF Personal Communications", Technical Digest, IEEE Custom Integrated Circuits Conference, Santa Clara, CA, May 1995, pp. 83-90.

[2] M. Thamsirianunt, T. A. Kwasniewski, "A $1.2 \mu \mathrm{m}$ CMOS Implementation of a Low-Power $900-\mathrm{MHz}$ Mobile Radio Frequency Synthesizer", Technical Digest, IEEE Custom Integrated Circuits Conference, San Diego, CA, May 1994, pp. 383-386.

[3] M. Soyuer, K. A. Jenkins, J. N. Burghartz, M. D. Hulvey, "A 3V $4 \mathrm{GHz}$ nMOS Voltage-Controlled Oscillator with Integrated Resonator", Technical Digest, IEEE International Solid-State Circuits Conference, San Francisco, CA, February 1996, pp.394-395.

[4] R. T. Howe, "Recent Advances in Surface Micromachining", Technical Digest, The 13th Sensor Symposium, 1995, pp. 1-8.

[5] L. J. Hornbeck, "Deformable-Mirror Spatial Light Modulators", in Spatial Light Modulators and Application III, Proceeding of The SPIE - The International Society For Optical Engineering (1990), vol. 1150, San Diego, CA, August 1989, pp. 86-102.

[6] C. W. Storment, D. A. Borkholder, V. Westerlind, J. W. Suh, N. I. Maluf, and G. T. A. Kovacs, "Flexible, Dry-Released Process for Aluminum Electrostatic Actuators", in Journal of Microelectromechanical System, vol. 3, No. 3, September, 1994, pp.90-96.

[7] A. P. Pisano, Y. H. Cho, "Mechanical Design Issues in Laterally-Driven Microstructures", Sensor and Actuators, A21-A23, 1990, pp. 1060-1064. 